\title{
Contribution à l'amélioration de la compréhension et de la traduction des adjectifs composés en classe de langue de spécialité
}

Mourad Boughedaoui

\section{OpenEdition}

\section{Journals}

Édition électronique

URL : http://journals.openedition.org/asp/3383

DOI : 10.4000/asp.3383

ISSN : 2108-6354

Éditeur

Groupe d'étude et de recherche en anglais de spécialité

\section{Édition imprimée}

Date de publication : 1 décembre 1997

Pagination : 525-541

ISSN : 1246-8185

\section{Référence électronique}

Mourad Boughedaoui, « Contribution à l'amélioration de la compréhension et de la traduction des adjectifs composés en classe de langue de spécialité », ASp [En ligne], 15-18| 1997, mis en ligne le 06 mars 2013, consulté le 30 avril 2019. URL : http://journals.openedition.org/asp/3383 ; DOI : 10.4000/ asp.3383

Ce document a été généré automatiquement le 30 avril 2019.

Tous droits réservés 


\title{
Contribution à l'amélioration de la compréhension et de la traduction des adjectifs composés en classe de langue de spécialité
}

\author{
Mourad Boughedaoui
}

1 Une des caractéristiques des langues de spécialité scientifique, technique et technologique est le besoin sans cesse croissant de former et d'intégrer dans leur vocabulaire un nombre relativement important de nouvelles unités lexicales. Cette néologie systématique touche essentiellement trois catégories syntaxiques: le nom, l'adjectif et le verbe. Si les néologismes nominaux servent à dénoter de nouvelles techniques ou de nouveaux concepts, les néologismes adjectivaux servent à en préciser davantage les contenus. Le moyen le plus privilégié semble être la composition adjectivale, qui constitue un procédé plein de vitalité, à en juger par le nombre des adjectifs composés dans les textes scientifiques. Elle constitue un processus important et multiforme qui permet de caractériser avec une plus grande précision et concision les syntagmes nominaux.

2 Dans une série d'articles publiés dans les Cahiers de l'APLIUT (Boughedaoui : 1993, 1995, 1996), nous avons tout d'abord axé notre recherche sur la dynamique qui sous-tend la lexigénèse des adjectifs composés dans un corpus de textes en anglais informatique. Cette recherche a permis de mettre en évidence le rôle des paires collocationnelles comme procédé générateur des adjectifs composés. Nous avons ensuite inventorié les principaux types d'adjectifs composés en fonction de leur fréquence d'emploi. Cet inventaire, établi à partir d'un corpus, a servi à l'étude des relations sémantiques qu'entretiennent entre eux les éléments des adjectifs composés. Tout au long de cet article, le premier et le second terme d'un adjectif composé seront désignés respectivement par «élémentspécificateur » et "élément-base ». La comparaison des types d'adjectifs composés recensés et ceux relevés par Tournier $(1985: 122)$ a permis de dégager un point supplémentaire concourant à la divergence entre la langue générale et la langue de 
spécialité, à savoir l'emploi contrasté de certains types d'adjectifs composés par rapport à d'autres.

3 Au terme de ces recherches, et dans un souci d'efficacité pédagogique, il nous est apparu indispensable de nous intéresser à la manière dont nos apprenants réagiraient face à ce phénomène. À cet effet, nous avons élaboré un test afin de procéder à cette évaluation.

\section{Élaboration du test et objectifs}

4 Le public visé par cette étude sont les élèves de première et de deuxième année du Département Statistiques et traitement informatique des données (STID) ${ }^{1}$ à l'IUT de Paris 5. L'objectif de ce test est essentiellement centré sur la capacité de nos apprenants à interpréter et à traduire les adjectifs composés relevés dans des textes authentiques. Ces textes ont été sélectionnés dans la revue Business Week, que nous utilisons fréquemment comme source d'approvisionnement en textes de statistique descriptive. Nous avons extrait 591 adjectifs composés à partir de quelques articles parus dans deux numéros de cette revue ${ }^{2}$. Les adjectifs composés ont été regroupés en fonction de leur appartenance à une même structure et ont été classés en fonction du pourcentage de leur fréquence d'emploi. Les exemples d'adjectifs composés sélectionnés pour ce test constituent dixneuf (19) types différents. Le test a été soumis à quatre groupes d'élèves de première année et quatre de deuxième année, soit un total de 106 étudiants. Compte tenu du nombre et de la variété des types, nous les avons répartis en quatre tests distincts composés chacun d'une vingtaine d'adjectifs composés (voir exemplaire en annexe 1).

Lors du déroulement du test, tous les termes pouvant présenter des difficultés aux étudiants sont expliqués soit dans les énoncés du test, soit oralement par l'enseignant ${ }^{3}$. L'objectif du test n'est pas centré sur la maîtrise du vocabulaire mais sur l'aptitude des apprenants à interpréter les relations sémantiques entre les éléments des adjectifs composés proposés. Tous les adjectifs composés traités dans le test sont présentés en contexte dans les phrases dans lesquelles ils apparaissent.

\section{Traitement des données et analyse des résultats}

6 Afin de procéder à la correction des traductions, nous avons distingué quatre catégories d'adjectifs composés :

7 a) les adjectifs composés ayant une entrée dans le dictionnaire (général ou de spécialité) [ex. : high-profile = très en vue, influant]

8 b) les adjectifs composés dont un seul élément (plus fréquemment l'élément-base) sert à la génération d'adjectifs composés [ex. : -related = (qui est) lié à... (repris de aging-related problems)]

9 c) les adjectifs composés que l'on retrouve dans le dictionnaire sous forme de noms composés ou de paires collocationnelles (dont les éléments ne sont pas reliés par un trait d'union) [ex. : junk mail = imprimés publicitaires non sollicités, adressés par la poste]

10 d) et enfin les adjectifs composés n'ayant pas d'entrées dans le dictionnaire et nécessitant une interprétation en relation avec le contexte [ex.: multiple-intelligence $=$ fondé sur l'existence de plusieurs intelligences]. 
11 On peut considérer que les adjectifs composés entrant dans les catégories a) b) et c) relèvent de la langue et que ceux de la catégorie $\mathrm{d}$ ) relèvent du discours. Ces derniers sont des adjectifs composés non répertoriés, constituant une classe ouverte à la création de nouvelles unités sans pour autant multiplier le vocabulaire de base.

$12 \mathrm{Au}$ terme de la correction des 106 copies du test, nous avons obtenu les résultats présentés dans les tableaux 1 à 4 représentant les quatre séries de tests.

Tableau 1. Récapitulatif des résultats du test 1 (exprimés en \%) :

\begin{tabular}{|l|l|}
\hline Typologie des adjectifs composés & Total élèves : $\mathbf{2 4}$ \\
\hline Adj + N & $37.50 \%$ \\
\hline $\mathrm{N}+\mathrm{N}$ & $33.34 \%$ \\
\hline $\mathrm{N}+\mathrm{V}-\mathrm{ed}$ & $38.33 \%$ \\
\hline V-ing + N & $16.67 \%$ \\
\hline \hline $\mathrm{N}+\mathrm{Adv} / \mathrm{Adj}$ & $39.59 \%$ \\
\hline
\end{tabular}

Tableau 2. Récapitulatif des résultats du test 2 (exprimés en \%) :

\begin{tabular}{|l|l|}
\hline Typologie des adjectifs composés & Total élèves : 23 \\
\hline Adv + V-ed & $42.51 \%$ \\
\hline $\mathrm{N}+$ Adj & $45.47 \%$ \\
\hline $\mathrm{N}+\mathrm{V}$-ing & $43.18 \%$ \\
\hline $\mathrm{V}^{\circ}+$ Adv & $17.55 \%$ \\
\hline Pref + V-ed & $38.07 \%$ \\
\hline
\end{tabular}

Tableau 3. Récapitulatif des résultats du test 3 (exprimés en \%) :

\begin{tabular}{|l|l|}
\hline Typologie des adjectifs composés & Total élèves : 25 \\
\hline Pref + N & $41.22 \%$ \\
\hline Adv + Adj & $33.72 \%$ \\
\hline Adv/Adj + V-ing & $19.42 \%$ \\
\hline Prep + N & $33.44 \%$ \\
\hline
\end{tabular}


V-ed $+\mathrm{N}$

$11.70 \%$

Tableau 4. Récapitulatif des résultats du test 4 (exprimés en \%) :

\begin{tabular}{|l|l|}
\hline Typologie des adjectifs composés & Total élèves : 34 \\
\hline Adj + Adj & $42.97 \%$ \\
\hline Adj + N-ed & $29.97 \%$ \\
\hline V-ed + Adv & $43.53 \%$ \\
\hline N + N-ed & $44.05 \%$ \\
\hline
\end{tabular}

13 Ces tableaux permettent de recenser les structures qui posent des problèmes d'interprétation à nos étudiants. Nous les reclassons dans le tableau 5 par ordre croissant des pourcentages.

Tableau 5. Récapitulatif des structures en fonction des pourcentages croissants

\begin{tabular}{|c|c|c|c|c|c|}
\hline 10 à $20 \%$ & 20 à $30 \%$ & \multicolumn{2}{|c|}{30 à $40 \%$} & \multicolumn{2}{|c|}{40 à $50 \%$} \\
\hline V-ed + N $11.70 \%$ & Adj + N-ed 29.97\% & $\mathrm{N}+\mathrm{N}$ & $33.34 \%$ & Pref $+\mathrm{N}$ & $41.22 \%$ \\
\hline V-ing + N $16.67 \%$ & & Prep + N & $33.44 \%$ & Adv + V-ed & $42.51 \%$ \\
\hline $\mathrm{V}^{\circ}+\mathrm{Adv} \quad 17.55 \%$ & & $A d v+A d j$ & $33.72 \%$ & Adj + Adj & $42.97 \%$ \\
\hline Adv +V-ing $19.42 \%$ & & $\operatorname{Adj}+\mathrm{N}$ & $37.50 \%$ & $N+V$-ing & $43.18 \%$ \\
\hline & & Pref + V-e & d $38.07 \%$ & V-ed + Adv & $43.53 \%$ \\
\hline & & $\mathrm{N}+\mathrm{V}$-ed & $38.33 \%$ & $\mathrm{~N}+\mathrm{N}$-ed & $44.05 \%$ \\
\hline & & $\mathrm{N}+\mathrm{Adv}$ & $39.59 \%$ & $N+A d j$ & $45.47 \%$ \\
\hline
\end{tabular}

Parmi les exemples traités dans la première colonne, ceux qui ont posé le plus de difficultés sont les structures où l'élément-base est un nom précédé d'un participe passé ou d'un participe présent comme élément-spécificateur. Ceci dénote une méconnaissance par les apprenants de l'emploi de ces deux formes participiales comme déterminant lexical placé en épithète devant un nom. Cet aspect est d'autant plus prépondérant qu'il s'agit de formes participiales de verbes irréguliers. Des deux exemples présentés dans le test, la séquence cut-rate Macintosh computers contient un participe passé d'un verbe irrégulier, qui n'a pas été identifié en tant que tel, à en juger par les traductions des étudiants : "proportion de Macintosh en panne » ou «taux de coupure des ordinateurs Macintosh» (sic). Les formes participiales en -ing se prêtent mal elles aussi à l'interprétation du fait qu'elles peuvent correspondre suivant les cas soit à un adjectif 
verbal, soit à un gérondif. Dans les exemples proposés dans le test, existing-home, nursinghome et emerging-market, nursing est un gérondif constituant le premier élément d'un nom composé pouvant se gloser par home for nursing, alors que les deux autres sont des adjectifs verbaux pouvant se gloser respectivement par homes that already exist (as opposed to newly-built ones) et markets that are emerging (=expanding) today.

En ce qui concerne le troisième type, à savoir $\left[\mathrm{V}^{\circ}+\mathrm{Adv}\right]$, les erreurs de traduction semblent être dues à des confusions entre a) un verbe suivi d'une particule adverbiale et un nom suivi d'une préposition (comme le montre la traduction du syntagme add-on programs par « annonce sur les programmes » où il $\mathrm{y}$ a évidemment confusion des termes to add et $a d$ ), et b) les adverbes et les adjectifs (comme l'attestent les traductions de diehard fans par « mort violente des fans » ou alors « des fans de mort violente »)(sic). À ceci nous rajouterons l'inobservation par l'apprenant de la règle fondamentale de la traduction de composés nominaux et de certains composés adjectivaux qui consiste à remonter de la droite vers la gauche à partir du dernier terme.

Dans le dernier type d'adjectifs composés, la combinaison [Adv $+\mathrm{V}-\mathrm{ing}$ ], nous retrouvons la forme participiale en -ing mais cette fois-ci en tant qu'élément-base précédé d'un adverbe. Cette structure résulte essentiellement de la réduction/transformation de propositions relatives actives. Dans l'exemple fast-growing credit-card, l'adjectif composé fast-growing provient de la transformation de: a credit-card that is growing fast. En appliquant ce modèle de transformation, c'est-à-dire en développant l'adjectif composé en proposition relative active, les apprenants amélioreraient certainement leur approche de ce type d'adjectifs composés. En fait, les traductions des étudiants laissent transparaître une lecture de gauche à droite des syntagmes nominaux qui donne lieu à des traductions où le verbe est nominalisé. Dans la majorité des copies, les adjectifs composés slow-selling, long-lasting et fast-growing ont été traduits respectivement par " vente lente », « longue durée » et " rapide augmentation ».

Pour les adjectifs composés ayant un pourcentage de bonnes traductions situé entre 20 et $30 \%$, nous n'avons recensé qu'une seule combinaison : [Adv/Adj + N-ed]. L'élément-base de cette structure est nom suffixé en -ed. Nous avons relevé diverses interprétations des exemples proposés. Pour illustration, nous reprendrons quelques traductions données par les étudiants pour les syntagmes high-powered infrared laser et de open-ended country funds : « haut pouvoir d'infra-rouge/laser à haut pouvoir infra-rouge » et « l'ouverture de la fin des pays isolés/la fin d'une ouverture des fonds du pays " (sic). Ces quelques exemples montrent là encore d'une part que la lecture des structures propres à la composition nominale et adjectivale se fait de gauche à droite, et d'autre part que l'adjectif composé n'est pas perçu en tant qu'élément de modification du nom. Nous noterons pour cette combinaison adjectivale les risques de confusion qui peuvent survenir avec les cas où l'élément-base est une forme participiale en -ed d'un verbe régulier. La nature de l'élément-spécificateur peut dans ces cas aider à lever l'ambiguïté : si c'est un adjectif, l'élément-base est un nom (suffixé), et si c'est un adverbe, l'élémentbase est un verbe (au participe).

Nous traiterons les autres types d'adjectifs composés en mettant seulement en évidence les cas les plus pertinents pour notre étude. Pour le type $[\mathrm{N}+\mathrm{N}]$, caractérisé par des possibilités d'interprétation très variées, nous avons relevé le cas des deux adjectifs junkmail et consumer-electronics qui ont eu les pourcentages respectifs suivants : 16,67 \% (soit 4 bonnes réponses sur 24 ) et $12,50 \%$ (soit 3 bonnes réponses sur 24). Nous reprenons pour ces exemples quelques traductions des étudiants: «mauvais courrier/courrier qui 
fonctionne mal » et "les acheteurs en électronique/électronique des consommateurs » (sic). On remarque toujours les mêmes erreurs essentiellement dues à une mauvaise perception du double niveau de détermination qui s'opère au sein de l'ensemble du syntagme nominal : un premier niveau où le premier terme de l'adjectif composé modifie le second et un deuxième niveau où ces deux éléments (désormais déterminant unique) modifient à leur tour le déterminé(nom simple ou composé).

Parmi les exemples relevant de la combinaison [Prep + N], seul l'adjectif composé in-house, relevé de in-house doctors, a connu un faible pourcentage de $04 \%$ (soit 1 seule bonne réponse sur 25). Les étudiants, fortement influencés par l'association sémantique doctor+in thouse, ont donné l'inévitable traduction « docteurs/médecins à domicile ».

En ce qui concerne la formation [Adv+Adj], nous avons relevé les deux exemples longoverdue et near-bankrupt relevés dans long-overdue consolidation et near-bankrupt systems. Leurs pourcentages respectifs sont $0 \%$ et $16 \%$ ( 4 sur 25 ). La seule traduction proposée pour le premier a été "une longue consolidation" où le terme "overdue » a été tout simplement omis. Les erreurs de traduction du deuxième adjectif composé ont été dues à une lecture linéaire directe et à la perception de «near » comme adjectif et non comme adverbe : « la récente faillite du système » et « système en cas de faillite » (sic).

Parmi les exemples représentant la structure [Adj $+\mathrm{N}$ ], le seul cas qui a posé des difficultés de traduction a été one-stop repris de one-stop shopping. Il a eu un pourcentage de $16,67 \%$ (soit 4 sur 24 ) avec des erreurs de traduction du genre « un coup d'arrêt aux ventes/un marché de plafond/magasin spécialisé » (sic).

Pour les structures qui restent à étudier, nous continuerons à concentrer notre attention sur celles qui ont posé de sérieux problèmes d'interprétation. La combinaison [N + V-ed], générée dans la majorité des cas à partir de la transformation d'une relative passive (Boughedaoui 1993 : 49), est mal perçue quand il s'agit plus particulièrement du participe passé d'un verbe irrégulier. Nous reprendrons, pour illustration, quelques traductions typiques données par les apprenants pour les adjectifs composés custom-made et state-run qui ont eu les pourcentages respectifs de 29,17 ( 7 sur 24 ) et 33,33 ( 8 sur 24). Ces derniers ont été repris de custom-made solutions et state-run monopolies et ont été traduits par « solutions pour se faire une clientèle/solutions imaginées par la clientèle » et « course des pays pour le monopole/monopole des États-Unis».

Des deux exemples de la combinaison [N+Adv], l'adjectif composé text-only a eu 33,33 \% (8 sur 24) de traductions acceptables. Nous citerons deux erreurs de traduction « texte seul et format normal » et « texte à format unique » (sic).

Concernant la structure [Pref $+\mathrm{N}$ ], l'adjectif composé multi-purpose de la séquence nominale multi-purpose vehicle a donné lieu à des traductions erronées liées aussi à une lecture linéaire et dont nous reprenons ces deux exemples « une multitude de choix de véhicules » et « une multitude d'objectifs sur un véhicule » (sic).

Pour la combinaison [Adv $+\mathrm{V}$-ed], qui résulte généralement de la transformation de propositions relatives passives et où le verbe est complété par un adverbe de manière, les difficultés concernent surtout les formes participiales des verbes irréguliers. Sur les cinq adjectifs composés, un seul renfermait un participe passé irrégulier, à savoir clear-cut repris de la séquence clear-cut evidence. Il a été diversement interprété : « évidence claire et coupée/évidence tirée au clair/évidence coupée nette ».

26 Les exemples de la structure [Adj + Adj] ont aussi donné lieu à des erreurs de traduction. L'adjectif composé squeaky-clean de la séquence squeaky-clean loan portfolio a eu un 
pourcentage de 08,82 (soit 3 sur 34). Le deuxième adjectif composé, spanking-white tiré de spanking-white coats a eu $08,82 \%$ (5 sur 34$)$. Quant à red-hot de la séquence red-hot rivalry, il a été traduit de façon inadaptée par «rivalité sanglante/rivalité chauffée au rouge/la chaleur rouge de la rivalité » (sic).

La structure [N + V-ing] correspond à des transformations de propositions relatives actives (Boughedaoui 1993: 50). L'élément-spécificateur est en fait le complément de la forme participiale en -ing. Nous remarquons que dans la traduction des adjectifs composés proposés les formes en -ing sont généralement assimilées à des substantifs ou inadéquatement interprétés. Les exemples que nous reprenons sont rate-setting, cropspraying et money-losing des séquences rate-setting Federal Committee, crop-spraying airplanes et money-losing plant. Nous en proposons respectivement quelques traductions types: «taux de siège du Comité Fédéral/le taux fixé par le Comité Fédéral»; "compagnie aérienne pour arroser les plantes/des avions versant des produits agricoles » et enfin « plante à faire perdre de l'argent/le plant de l'argent manquant » (sic).

Les exemples de la structure [v-ed + Adv], à l'exception de built-in, ont été correctement traduits. Ce dernier, repris de la séquence built-in floppy drive, a donné lieu à des erreurs de traduction telles que « un driver déjà construit/un driver construit en floppy » [sic].

La combinaison [ $\mathrm{N}+\mathrm{N}-\mathrm{ed}$ ], où l'on retrouve comme élément-base un nom suffixé en -ed, a été relativement bien traduite. Pour le test nous avions les adjectifs composés cementwalled et ring-shaped des séquences cement-walled factories et ring-shaped seals. Le premier a eu un pourcentage de 69,23 contre 30,77 pour le deuxième. Cette différence s'explique par le fait que les apprenants ont traduit cement-walled par « intuition » en se fondant sur le rapprochement sémantique entre cement et wall, le premier servant généralement à la construction du deuxième. Cependant, nous avons noté quelques contresens dans la traduction de cet adjectif composé: "industries en ciment/usines de ciment». En revanche, le deuxième adjectif composé ring-shaped a donné lieu à un plus grand nombre d'erreurs de traduction dont nous reprenons quelques-unes à titre d'illustration: " anneau du joint d'étanchéité/joint serti d'une bague ».

30 Enfin, la dernière combinaison du test composée de [N + Adj] a connu les pourcentages les plus élevés, bien que toujours en dessous de la moyenne. Sur les cinq exemples traités dans le test, seul l'adjectif composé error-prone a connu un pourcentage élevé. En revanche, nous avons enregistré des erreurs d'interprétation pour les adjectifs composés defect-free, subject-specific et wattage-hungry relevés dans les séquences respectives defectfree vehicle, subject-specific exams et fairly wattage-hungry. Pour le premier nous avons même eu des traductions diamétralement opposées: "véhicules défectueux/véhicules plein de défauts/libre défaut du véhicule ». Concernant le deuxième, les erreurs ont été dues à des inversions de structure "sujet spécifique d'examen/examen spécifique du sujet ». Par exemple, l'adjectif composé wattage-hungry a été traduit par « consommation équitable de watts/consommation de watts raisonnable ».

31 Dans l'ensemble l'analyse des résultats obtenus a fait ressortir assez clairement les difficultés rencontrées par nos apprenants dans la traduction des divers types d'adjectifs composés. Ceci peut s'expliquer d'une part par la rareté, voire l'inexistence, de ce phénomène dans leur langue maternelle et d'autre part par le fait que peu d'attention est accordée à la composition adjectivale dans les ouvrages pédagogiques. Cependant, en tenant compte de la fréquence d'emploi et de la variété des types d'adjectifs composés dans les textes scientifiques et techniques, leur étude est vivement préconisée en classe de langue de spécialité. Ils constituent en fait un support non négligeable à la 
compréhension d'un autre phénomène propre au discours scientifique et technique : les noms composés en chaîne. Leur introduction dans un cursus d'étude se ferait de façon graduelle, brève et variée afin de couvrir les types les plus fréquemment utilisés. En travaillant sur des séries d'exercices appropriés, nos apprenants seraient régulièrement exposés à ce phénomène. Ils développeraient ainsi leurs capacités à appréhender et à interpréter avec un maximum d'autonomie, d'efficacité et de spontanéité les différents types d'adjectifs composés.

\section{Adjectifs composés de langue et de discours}

Nous avons pu mettre en évidence au terme de notre étude l'existence de deux catégories d'adjectifs composés (voir figure 1). La première concerne les adjectifs composés indépendants du contexte et appartenant à la langue. La deuxième concerne ceux qui dépendent étroitement du contexte dans lequel ils apparaissent et relèvent ainsi du discours. Pour la première catégorie, il s'agit de séquences lexicalisées dont les constituants ont été progressivement «soudés » ensemble par l'usage pour former une suite indissociable exprimant une notion unique. Pour ce qui concerne la deuxième catégorie, il s'agit plutôt de séquences fortuites dont les constituants sont juxtaposés de façon ad hoc afin de répondre à des besoins ponctuels de détermination. Il est cependant possible à ces constituants d'évoluer vers la lexicalisation à mesure que s'accroît leur fréquence d'emploi.

\section{Élaboration des exercices}

La rareté des exercices traitant des adjectifs composés nous amène, nous enseignants en langue de spécialité, à concevoir nos propres exercices pour répondre aux besoins de nos apprenants. Si la composition nominale connaît un regain d'intérêt dans les ouvrages pédagogiques en anglais de spécialité, il n'en est pas de même pour la composition de l'adjectif. C. Lassure (1990: 55) a été un des rares auteurs à consacrer plusieurs parties de son ouvrage à l'étude d'adjectifs composés. Bien que quelques types seulement y aient été traités, ils l'ont été de façon exhaustive et riche en exemples et exercices. Nous retrouvons à travers d'autres ouvrages de brèves références aux adjectifs composés. J. Upjohn et al. (1991: 111) consacrent une section à l'étude de la composition nominale et adjectivale. Pour cette dernière, seulement trois types y ont été introduits sous forme d'exemples. S. Bosworth et al. (1992 : 181) dans leur chapitre 3 consacrent une partie à l'étude des noms composés. Les auteurs font référence indirectement aux adjectifs composés en parlant du rôle du trait d'union dans les noms composés. M. Brookes et al. (1993 : 53) traitent aussi de la composition nominale et parlent de l'adjectif composé dans un court passage que nous reproduisons ci-après :

A very compact noun phrase structure is often made even more compact by the addition of compound adjectives which are sometimes, but not always, separated by a hyphen:

software-controlled security features (=security features that are controlled by software)

this disk-based system (=this system that is based on disks)

$\mathrm{Au}$ terme de notre test sur les adjectifs composés, nous constatons que la difficulté réside dans la variété des types. Faute de pouvoir intégrer dans un cursus tous les types 
recensés, il faut se limiter aux types les plus fréquemment employés. Il s'agit en fait de faire prendre conscience à nos apprenants de l'importance de ce phénomène et de les préparer à y faire face de la façon la plus pragmatique possible. Le meilleur moyen d'y arriver consiste à mettre en place des exercices appropriés, variés et aptes à faire acquérir progressivement la maîtrise du processus de la composition adjectivale, tant sur le plan de l'interprétation que sur celui de la production. Les exercices que nous proposons dans cette étude sont fondés sur deux approches différentes selon qu'il s'agisse de la première catégorie d'adjectifs composés ou de la deuxième.

Figure 1. Deux catégories d'adjectifs composés

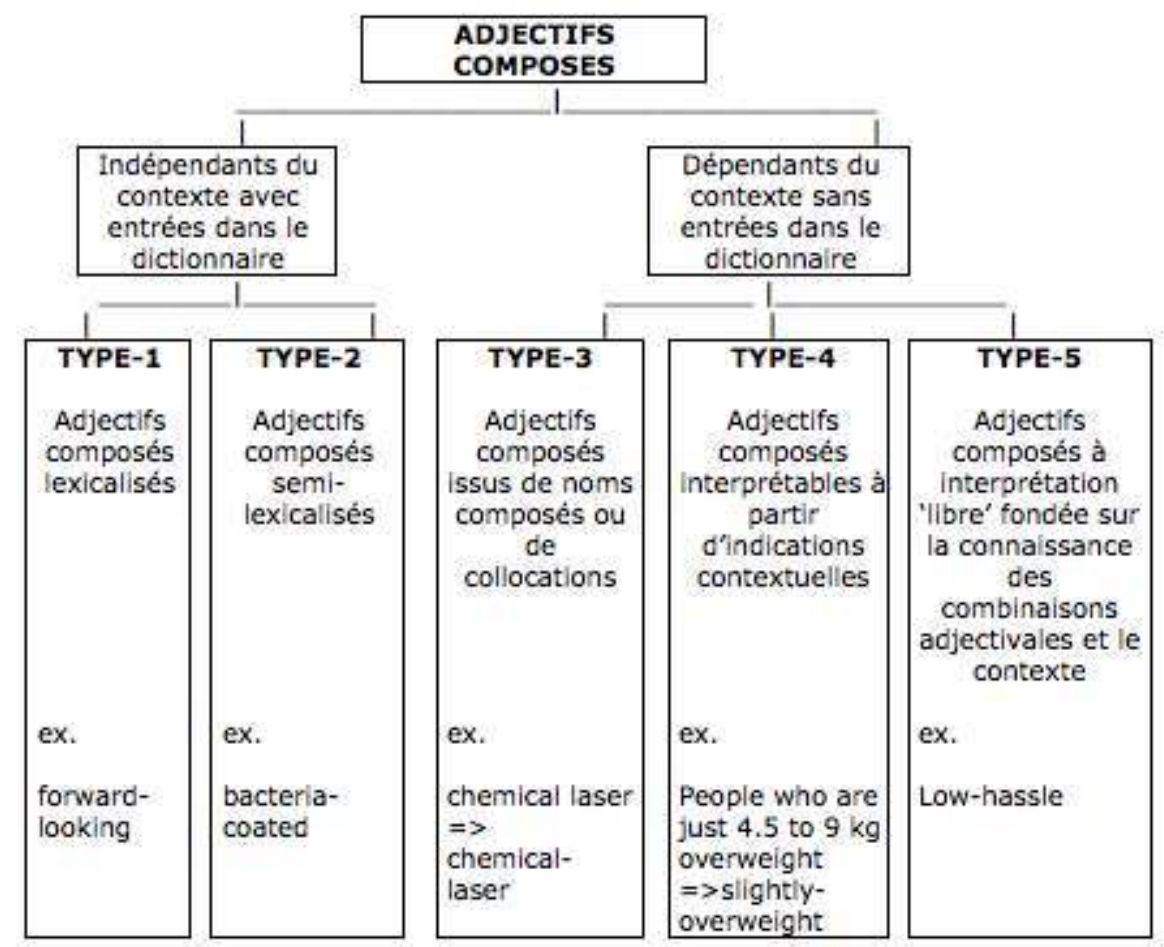

Pour cette catégorie d'adjectifs composés, représentés par les types 1 et 2 du diagramme ci-dessus, nous proposons des exercices complémentaires qui ont pour but l'assimilation des mécanismes régissant la formation des adjectifs composés. Il est essentiel pour l'apprenant de prendre conscience que si en anglais nous avons un adjectif composé de deux termes, il ne sera pas systématiquement traduit par deux autres termes en français. Les signifiés n'étant jamais exactement superposables, il ne peut y avoir de traduction mot à mot. C'est la recherche d'équivalences qui guidera l'apprenant dans la sélection des termes les plus appropriés.

\section{Exercices pour les adjectifs composés indépendants du contexte}

a) Combine the words from lists A and B to make compound adjectives that correspond to the French translations given below:

\begin{tabular}{|l|l|}
\hline A & B \\
\hline
\end{tabular}




\begin{tabular}{|c|c|}
\hline custom & run \\
\hline long & looking \\
\hline state & ended \\
\hline open & made \\
\hline forward & lasting \\
\hline 1. Prévisionnel & \\
\hline 2. Sans limite de durée ; à deux ouvertures & $\mid \ldots$ \\
\hline 3. Sur mesure & \\
\hline 4. Durable & \\
\hline 5. D'état, géré par l'état & |... \\
\hline
\end{tabular}

L'apprenant peut à ce stade de l'exercice utiliser le dictionnaire pour procéder à la vérification des combinaisons qu'il a obtenues. Ceci lui permettra d'infirmer ou de confirmer le choix qu'il a opéré. Comme première variante complémentaire à cet exercice, on peut aussi demander à l'apprenant d'insérer chacun des adjectifs composés qu'il a obtenus dans des phrases qui lui sont proposées comme contextes potentiels.

b) Fill in the blanks with one of the above compound adjectives:

1. If your company faces communication challenges, no one can offer you more solutions than Deutsche Telecom.

2. All sectors revised up spending plans, a key indicator.

3. Scientists unsuccessfully urged the Food and Drug Administration to delay its approval because of worries over changes in animals.

4. Market experts attribute the discounts on the funds to poor performance and country funds that boast low expenses and trade on U.S. exchanges.

5. When Germany and Britain began dismantling monopolies, the U.S.

Baby Bells immediately jumped into the breach.

On peut évidemment adapter les phrases en fonction du niveau des apprenants et de leur domaine de spécialité. On peut par exemple présenter un contexte plus réduit avec seulement des noms devant être modifiés par les adjectifs composés reconstitués :

c) Insert the above compound adjectives in the blank spaces:

1. companies

2. a(n) _-_-_-_ person

3. a(n) --_-_-_ meeting / tube

4. -_-_-_-_-_-_ clothes

5. $\mathrm{a}(\mathrm{n})$ _-_-_-_-_ link

40 Un autre type d'exercice, fondé sur la reconstitution d'adjectifs composés à partir de propositions relatives à réduire, peut être proposé aux apprenants afin de leur permettre de mieux percevoir les transformations en amont d'un adjectif composé et de mieux adapter leur traduction :

d) Make up compound adjectives that would result from the reduction of the

following relative clauses: 
1. That is run by the state:

2. That looks forward:

3. That is open on both ends :

4. That is made according to the customer's needs :

5. That lasts for a long time :

Une variante à cet exercice consisterait à demander aux apprenants de proposer une traduction pour chacun des adjectifs composés obtenus après réduction. Le dictionnaire peut être utilisé par l'apprenant pour vérifier les résultats auxquels il sera parvenu.

Pour traiter le type 2, les adjectifs semi-lexicalisés, on peut concevoir des exercices de traduction qui montreraient la récurrence de certains termes et la possibilité qu'ils offrent pour générer de nouvelles séquences adjectivales (voir ci-dessous).

a) Translate the following compound adjectives (what do you notice? Can you give examples of your own?):

\begin{tabular}{|c|c|}
\hline 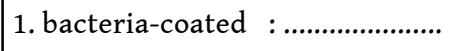 & 7. job-related : .............................. \\
\hline 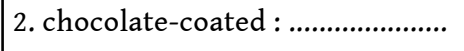 & 8. aircraft-related : .............................. \\
\hline 3. gold-coated : .......................... & 9. railway-related : ............................... \\
\hline 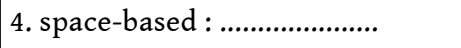 & 10. ring-shaped $\quad: .$. \\
\hline 5. office-based : ........................... & 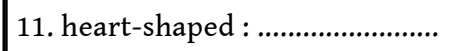 \\
\hline 6. Washington-based : .......................... & 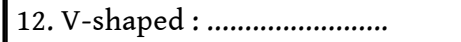 \\
\hline
\end{tabular}

\section{Exercices pour les adjectifs composés dépendant du contexte}

En ce qui concerne cette catégorie d'adjectifs composés correspondants aux types 3, 4 et 5 du diagramme et dépendants étroitement du contexte, les exercices à proposer serviront de support parallèle à la compréhension de textes. Du fait que ces adjectifs n'ont pas d'entrées dans le dictionnaire, l'apprenant doit apprendre à repérer sur l'aire du texte toute indication ou développement pouvant contribuer à leur interprétation. Ceci concerne les adjectifs composés qui sont soit dérivés de paires collocationnelles précédemment introduites dans le même texte (type-3), soit explicités par des indications dans le texte (type-4). Nous étudierons ces deux types à travers des exemples repris des textes du corpus.

Les adjectifs composés que nous avons choisis pour illustrer le premier type sont existinghome (prices), adjustable-rate (mortgages), packaged-goods (companies), et chemical-laser (system). Pour chacun, nous donnerons ci-après le passage contenant la collocation ayant servi à le générer : "...most economists were expecting the bellwether U.S. housing industry to slow significantly by late summer. Instead, sales of new and existing homes have remained strong ... . " pour le premier adjectif composé et, toujours dans le même texte, le passage " And at $6 \%$ or so, adjustable rates are running two-and-a-half percentage points below conventional mortgage rates » comprenant la collocation du deuxième adjectif. Les passages renfermant les paires collocationnelles pour les deux autres adjectifs composés sont respectivement 
" ... outside packaged goods, the attack on complexity is gathering force in the auto industry. " et "The potential of chemical lasers to serve as defensive shields ... ». Nous remarquons que pour ces exemples une seule occurrence de la paire collocationnelle suffit pour engendrer un adjectif composé. En revanche, une fois que l'adjectif composé est formé, il peut servir à préciser le contenu sémantique de différents termes dans le texte. Tel est le cas de packaged-goods que nous retrouvons successivement dans «packaged-goods companies / packaged-goods marketing system ", ou encore de l'adjectif composé "multiple-intelligence " que nous retrouvons dans les séquences «multiple-intelligence theory/multiple-intelligence precepts/multiple-intelligence tests. » Les adjectifs composés peuvent être aussi issus de paires collocationnelles ayant plusieurs occurrences dans le texte. Ce sont généralement des collocations thématiques tenant lieu de mots-clés. Nous étudierons les exemples suivants : emerging-market (stocks), junk-mail (programs) et smart-well (components). Dans le texte d'où a été repris le premier composé adjectival, nous avons relevé les passages suivants : " Emerging markets : it pays to be picky", "Value investors these days must work hard to find true bargains in emerging markets ", « Emerging markets are still a great long-term story ", "The great long-term story, of course, is the classic argument for buying into emerging markets ", «... $10 \%$ in most emerging markets », et " That's why value investors are being super-picky in emerging markets. » Les passages concernant le deuxième adjectif composé sont : «... but the junk E-mail is still coming ", « Nobody can say precisely how big junk E-mail is ... », « Even as marketers catch on to junk E-mail, they face a backlash ", " even some pioneers of junk E-mail concede that there's a risk ... », et "The rapid expansion of targeted junk E-mail worries privacy advocates ». Pour le dernier exemple, on retrouve dans le texte les passages : «Companies working on the new gear for 'smart wells' range from ... ", " while smart wells spout oil, they also spout data ... » ou encore ce passage, très révélateur du sens à accorder à cet adjectif composé, qui relate les caractéristiques d'un nouveau procédé appelé à révolutionner la technique des forages : " A Well with Brains:[...] So oil companies are testing 'smart' valves that shut off the bad sources while letting the good ones keep producing ». Les séquences soulignées constituent en fait la clé qui donne accès au sens de ce composé adjectival. En ce qui concerne les exercices, il s'agit de faire travailler en amont les apprenants sur les collocations en question (p. ex. détermination des catégories syntaxiques, analyse des relations possibles entre les constituants et traduction à l'aide du dictionnaire) avant d'analyser leur rôle en tant qu'adjectifs composés épithètes placés avant les noms qu'ils qualifient.

Pour revenir au type-4 du diagramme, les adjectifs composés dont l'interprétation dépend étroitement d'informations contenues dans le texte, nous étudierons les cas de bacteria-coated (root), slightly-overweight (women), aging-related (problems), et enfin multipleintelligence (theory). Il s'agit de retrouver pour ces adjectifs composés les données contextuelles contribuant directement ou indirectement à leur interprétation. Celles-ci sont fréquemment liées à la thématique centrale du texte. La relation entre bacteria-coated et root est explicitée par le passage suivant, pris dans la première phrase du même texte : "Agriculture Dpt. microbiologists have managed to protect cucumber roots from fungal rot by coating seeds with beneficial bacteria. ». Pour le deuxième exemple, la relation entre slightlyoverweight et women est explicitée par le passage « Redux is going to be taken by people who are just 4.5 to $9 \mathrm{~kg}$ overweight » et par les séquences «seriously obese people » et «seriously overweight clients » qui sont en fait les contraires! Les indications que l'on peut recueillir sont parfois plus nombreuses et plus variées. C'est en effet le cas avec les deux autres exemples. Concernant la séquence "aging-related problems", nous notons ci-après les passages contribuant à son élucidation : "Today, scientists are still looking for antidotes to 
aging ... ", "The National Institute on Aging (NIA) has launched a program with a dozen or so laboratories to search for longevity genes", "... they will help prevent diseases of aging and increasing life span ", "Rather, the goal of this research is to increase individual longevity and to delay diseases associated with aging ", ou encore "... researchers have discovered a half-dozen genes that directly affect longevity ». Nous voyons qu'un seul adjectif composé peut couvrir tout le champ thématique d'un texte donné. Comme exercice, on peut proposer à l'apprenant de développer l'adjectif composé à étudier (en s'aidant s'il le faut du dictionnaire) avant de rechercher dans le texte toutes les indications qui concourent à sa compréhension. Pour la deuxième séquence, multiple-intelligence theory, nous avons relevé les indications suivantes: "... intelligence is'nt one thing but many ", "... there are at least eight different kinds of human intelligence ", "... to prove the existence of his eight separate intelligences ... », «... Gardner's ideas about multiple intelligences spring from his work beginning two decades ago with gifted children and victims of brain damage ", et "Gardner believes that people possess intelligences in varying degrees, that it's possible to hone them and that they'll wither with lack of use ". Là encore, nous pouvons travailler sur la thématique du texte à partir du sens accordé à cet adjectif composé. Ce type d'adjectifs composés à prédominance thématique ont plus de chance de se voir octroyer le « statut » de séquence lexicalisée identifiant un nouveau concept. Ils peuvent servir d'amorce à des exercices de compréhension et d'expression écrites.

Les adjectifs composés du type 5 sont les plus difficiles à interpréter du fait qu'ils n'ont ni entrées dans les dictionnaires ni indications complémentaires dans le texte. Seule la connaissance que peut avoir l'apprenant des différentes variations paradigmatiques propres à la composition de l'adjectif peut aider à la compréhension des diverses relations sémantiques entretenues par les termes du composé adjectival. C'est la dernière catégorie à introduire dans le cadre d'un cours. Nous proposons ci-après trois types d'exercices visant à faire acquérir par l'apprenant les mécanismes qui sont en amont de la formation des adjectifs composés et à lui assurer un maximum d'autonomie quant à leur interprétation. Le premier exercice est fondé essentiellement sur la reconstitution de syntagmes nominaux renfermant un ou deux adjectifs composés :

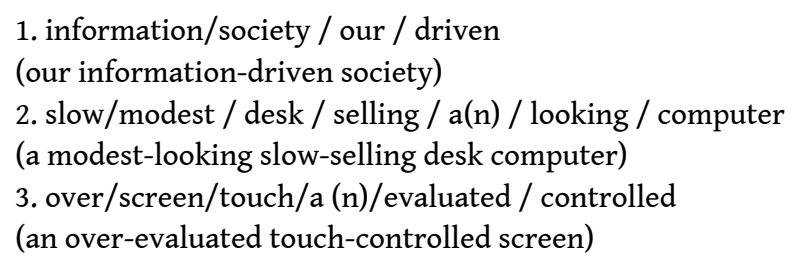

L'intérêt de cet exercice est qu'il donne lieu à d'intéressants débats sur l'acceptabilité des séquences adjectivales pouvant être éventuellement proposées par les apprenants. En effet, certaines combinaisons peuvent être difficilement interprétables du fait de l'inversion de leurs composants (p. ex. driven-information au lieu de information-driven pour le premier exemple) ou bien de l'incompatibilité des éléments (p. ex. modest-selling au lieu de modest-looking ou alors slow-looking au lieu de slow-selling pour le deuxième exemple). Pour des apprenants de niveau moyen, et afin de leur permettre de mieux orienter leur choix des termes, on peut proposer comme fil conducteur la traduction des adjectifs composés à retrouver.

Le deuxième exercice consiste à opérer des réductions de structures de postmodifications nominales (soulignées dans les exemples ci-dessous) en vue de générer des adjectifs composés : 
1. The Hawaiian Squid Euprymna Scolopes catches bacteria which produce light as it swims through the water. These bacteria can also pass their genes -which float freely inside the organisms that are composed of a single cell- to the cells of plants and animals.

Dans cet exercice, l'apprenant devra en premier lieu repérer à l'intérieur des phrases les structures de modification pouvant conduire, après transformation, à des adjectifs composés. Il procédera ensuite à ces transformations avant de réécrire le passage en y intégrant les adjectifs composés obtenus :

1. The Hawaiian Squid Euprymna Scolopes catches light-producing bacteria as it swims through the water. These bacteria can also pass their genes -which float freely inside single-celled organisms to the cells of plants and animals.

51 Le troisième et dernier exercice que nous proposons est fondé sur le développement d'adjectifs composés. L'objet de cet exercice est d'amener l'apprenant à rechercher les expansions syntagmatiques pouvant expliciter la relation sémantique existant entre les éléments de l'adjectif composé d'une part, et le terme déterminé d'autre part. Pour illustrer cet aspect, nous donnons quelques exemples suivis de leurs développements respectifs :

1. low-hassle buying: buying where the risk of hassle is low

2. overnight-delivery services: services specialized in overnight deliveries

3. subject-specific exams: exams that are specific to a (particular) subject

4. long-overdue consolidation: consolidation which has been overdue for a long

time

5. consumer-lending business: business concerned with lending to consumers

\section{Conclusion}

Dans notre étude, nous avons pu mettre en évidence les principales structures posant de sérieux problèmes de traductions à nos apprenants. Les performances relevées lors du test sont dans l'ensemble en dessous de la moyenne (avec 12 structures en dessous de $40 \%$ et seulement 7 entre 41 et $46 \%$ ). Comme nous l'avons souligné dans notre analyse, la composition de l'adjectif est un processus très productif et très varié dont il faut tenir compte dans notre enseignement. Cette diversité des types morphologiques entraîne des difficultés notables sur le plan de l'interprétation des relations sémantiques entre les éléments. Afin de préparer l'apprenant à surmonter ces difficultés, nous devons l'amener par le biais d'exercices appropriés à réfléchir avec pondération au sens à attribuer à chaque adjectif composé. La composition de l'adjectif constitue du triple point de vue de la variété des types, de la productivité et de la diversité des relations sémantiques un maillon incontournable dans l'élaboration des différents cursus propres aux langues de spécialités enseignées en IUT. 


\section{BIBLIOGRAPHIE}

Bosworth-Gerome, S., C. Ingrand \& R. Marret. 1992. Comprendre l'anglais scientifique et technique. Paris : Ellipses.

Boughedaoui, M. 1993. «L'adjectif composé en tant qu'élément de complexification du groupe nominal dans les textes informatiques ». Les Cahiers de l'APLIUT 13/1, 44-53.

Boughedaoui, M. 1995. " Les séquences collocationnelles et la dynamique de la composition adjectivale ». Les Cahiers de l'APLIUT 15/1, 47-57.

Boughedaoui, M. 1996. « Essai de catégorisation sémantique des adjectifs composés (1) ». Les Cahiers de l'APLIUT 16/1, vol XVI, 31-46.

Brookes, M. \& F. Lagoutte. 1993. English for the Computer World. Paris : Belin.

Lassure, C. 1990. TECH L'anglais des articles de presse et des documents techniques. Paris : Ellipses.

Tournier, J. 1985. Introduction descriptive à la lexicogénétique de l'anglais contemporain. Paris/Genève : Champion-Slatkine.

Upjohn, J., S. Blattes \& V Jans. 1991. Minimum Competence in Scientific English. Grenoble : Presses universitaires de Grenoble.

\section{ANNEXES}

\section{Annexe 1}

IUT PARIS V STID DEPARTMENT Year: FIRST - SECOND

Translate the nominal groups underlined in the sentences below.

1. In its latest report on 16,000 employers, Manpower says its index rose to a seasonallyadjusted level of 17 .

@ seasonally-adjusted level:

2. So far, however, it has been the makers of the fuzzily-focused devices who have been frustrated. [fuzzy = flou, confus; to focus = diriger, concentrer, fixer, mettre au point ] @ fuzzily-focused devices:

3. Greenspan seemed predisposed not to throw the Fed into the political debate by increasing rates without clear-cut evidence that the economy is strong enough to fuel inflation.

@ clear-cut evidence:

4. With the widened range of stocks available, investors now have the opportunity of building sectorially-balanced portfolios. [ to balance = équilibrer / portfolio = portefeuille ] @ sectorially-balanced portfolios:

5. Unless regulation is tightened, another financial scare could swiftly tarnish their hardearned reputation for free-market progress. [ to earn = gagner, mériter ]

@ hard-earned reputation: 
6. Another section helps students prepare for the subject-specific exams

@ subject-specific exams:

7. The Cincinnati-based company is leading a broad movement among marketers as executives realize that complexity alienates and generates expensive and error-prone operations. [prone=prédisposé, sujet à]

@ error-prone operations:

8. The bank of Japan may also push up its discount rate a smidgen from its record-low 0.5 \% to keep the Japanese economy moving ahead at a sustainable rate.

@ record-low $0.5 \%$ :

9. How can a hodge-podge of suppliers assemble a defect-free vehicle? [hodge-podge = amas confus ]

@ defect-free vehicle:

10. CD players are fairly wattage-hungry, and this unit can be powered by AA cells, by the lithium-ion battery, or by sharing an AC adapter with the computer.

@ fairly wattage-hungry:

11. The StudyWorks version focuses as much on test-taking techniques as it does on content.

@ test-taking techniques:

12. Being "in" puts you in the center of the decision-making processes of the new European institutions.

@ decision-making processes:

13. Tex Guthrie, a U.S. businessman, is the director for international sales at Ayres Corp., a small manufacturer of crop-spraying airplanes in Albany, Ga. [crop = produit agricole, culture, récolte]

@ crop-spraying airplanes:

14. The question before the rate-setting Federal Committee was one of the toughest it had faced in years.

@ rate-setting Federal Committee:

15. When GE bought the money-losing plant from France's Thompson in 1987, the unit was selling $75 \%$ of its production inside France.

@ money-losing plant:

16. The spoilers of this feel-good atmosphere could be the bond market and the Federal Reserve.

@ feel-good atmosphere:

17. Still, this developer of diagnostic and drug-delivery systems primarily transdermal patches, has some die-hard fans.

@ die-hard fans:

18. Add-on programs to Navigator, called plug-ins, are cumbersome to install. @ add-on programs:

19. The FDA plan may be a half-baked solution to the smoking problem.

@ half-baked solution: 
20. Modular mini-lines churn out sub-assembled sections. [to churn out=produire à la chaîne, en série ]

@ sub-assembled sections:

\section{NOTES}

1. IUT Paris 5,143 avenue de Versailles, 75016 Paris.

2. Business Week, September 9, 1996 et October 7, 1996.

3. Nous avons préféré éviter le recours au dictionnaire pour deux raisons essentielles : a) le nombre d'étudiants aurait exigé plusieurs dictionnaires et un temps de consultation plus long; $b$ ) les étudiants auraient recopié les traductions des adjectifs composés ayant une entrée dans le dictionnaire.

\section{RÉSUMÉS}

La composition adjectivale est un processus important et plein de vitalité qui contribue avec précision et concision à la caractérisation du nom. Par la grande variété des types et des relations sémantiques qui la caractérise, elle constitue un obstacle supplémentaire à la compréhension des groupes nominaux complexes. Nous avons tenté, à travers cette étude, d'évaluer l'aptitude de nos apprenants à comprendre et traduire les différents types d'adjectifs composés. Les résultats obtenus nous ont permis d'une part d'identifier les types qui posent le plus de difficulté et d'autre part de proposer une série d'exercices permettant à l'apprenant d'y faire face avec un maximum d'efficacité.

Adjectival compounding is a significant and productive process that contributes much accuracy and conciseness towards the characterization of nouns. Because of its great variety of types and semantic relationships, adjectival compounding constitutes a further hindrance to the comprehension of complex nominal groups. We have attempted, through this study, to assess our learners' ability to understand and translate the various types of compound adjectives. The results we have achieved have enabled us on the one hand to identify the types that pose a real problem and, on the other, to propose a series of exercises that will help the learner cope with compound adjectives in the most efficient way.

\section{INDEX}

Keywords : assessment test, adjective, compound adjective typology, ESP, exercise typology Mots-clés : adjectif, anglais de spécialité, test d'évaluation, typologie des adjectifs composés, typologie d'exercices 


\section{AUTEUR}

\section{MOURAD BOUGHEDAOUI}

Mourad Boughedaoui est maître de conférences à l'IUT de Paris 5, au Département Statistiques et traitement informatique des données. Ses recherches sont essentiellement orientées sur l'étude du phénomène de composition (nominale et adjectivale) au sein de groupes nominaux complexes. Il a publié une série d'articles sur les adjectifs composés dans les Cahiers de l'APLIUT et a proposé un autre article pour le volume 17/4, de juin 1998, intitulé « Comparative study of the distribution of adverb-adjective combinations with a special concern in English for statistics ».bougheda@iut.univ-paris5.fr 